\title{
Búsqueda de información en medicina basada en evidencia
}

\author{
Gabriel Rada G ${ }^{1}$, Maricarmen Andrade $\mathbf{A}^{1}$, \\ Virginia Leyton Sch ${ }^{1,2 a}$, Cecilia Pacheco $V^{1,2 a}$, \\ Esmeralda Ramos $\mathbf{R}^{2, a}$ \\ Search strategies \\ in evidence-based medicine
}

L a medicina ha sufrido un rápido proceso de cambio en los últimos años fundamentalmente debido a dos elementos: por un lado la presión asistencial y la escasez de tiempo derivada de ésta, son cada vez mayores y, por otra parte, la cantidad de información crece en forma exponencial. A modo de ejemplo, MEDLNE, la base de datos más importante actualmente, cuenta con doce millones de artículos y cada día se agregan cerca de 2.000 nuevos ${ }^{1}$. Es por esto que la necesidad de desenvolverse con facilidad en este torrente de información se ha vuelto muy importante, y se entiende como prioritario para la práctica de la medicina basada en evidencia (MBE). El advenimiento de la Internet, y su amplia disponibilidad, nos entregan las herramientas para acceder a este enorme conocimiento potencial y, lo que es más importante, trasladarlo a nuestra práctica clínica y a nuestros pacientes.

LA NECESIDAD DE FORMULAR UNA BUENA PREGUNTA CĹNICA

El primer paso, antes de iniciar una búsqueda, es definir exactamente lo que queremos buscar. Para esto es necesario formular una pregunta que contenga todos los elementos relevantes. En el caso de preguntas acerca de un determinado tratamiento, se requiere definir el tipo de pacientes en el cual estamos interesados, la intervención que deseamos evaluar y el outcome que nos interesa sea modificado por esta intervención ${ }^{2,3}$. En pocas palabras, formulamos una pregunta que pueda traducirse en una estrategia de búsqueda que nos permita encontrar lo que queremos, de la manera más rápida y efectiva.

\section{SENSIBILIDAD Y ESPECIFICIDAD DE UNA BÚSQUEDA}

Una vez definida la pregunta clínica, es necesario extraer de ella la información útil para desarrollar nuestra estrategia de búsqueda. Permanentemente debemos hacer un balance entre el tiempo que disponemos y el objetivo de la búsqueda. $\mathrm{Si}$ deseamos obtener toda la información que existe en relación a una pregunta, realizaremos una búsqueda amplia, usando términos genéricos y con pocos límites. Esta estrategia arrojará muchos

\footnotetext{
${ }^{1}$ Unidad de Medicina Basada en Evidencia, Pontificia Universidad Católica de Santiago de Chile. ${ }^{2}$ Biblioteca Biomédica, Sistema de Bibliotecas, Pontificia Universidad Católica de Santiago de Chile. aBibliotecaria
}

Correspondencia a: Dr. Gabriel Rada G. Unidad de Medicina Basada en Evidencia, Universidad Católica de Chile (e-mail: umbeuc@med.puc.cl). 
artículos, algunos serán relevantes y otros no, por lo tanto necesitaremos una cantidad de tiempo importante para encontrar la respuesta a nuestra pregunta. Esta estrategia corresponde a una búsqueda sensible. Por otro lado, si queremos obtener un número menor de artículos, pero de alta relevancia, que nos permitan responder a nuestra pregunta en forma más rápida, realizaremos una búsqueda específica. En este caso privilegiamos el tiempo por sobre la amplitud, reconociendo que existe la posibilidad de perder algunos artículos relevantes.

La enorme cantidad de información disponible y el poco tiempo que la mayoría de los clínicos tienen, conduce, generalmente, a realizar búsquedas específicas ${ }^{4}$.

\section{RECURSOS DE INFORMACIÓN DISPONIBLES EN LA INTERNET}

La posibilidad de encontrar lo que buscamos, en forma rápida y eficiente, dependerá en gran medida de una adecuada selección del recurso a emplear. Existen múltiples sitios médicos en donde se puede encontrar respuestas a preguntas médicas generales (preguntas básicas o de preparación) ${ }^{2}$. Bastará con utilizar textos que se basen en bibliografías adecuadas y que sean actualizados en forma frecuente. Dentro de los disponibles en formato electrónico se encuentran los siguientes:

- Up To Date (http://www.uptodate.com): un buen recurso, se actualiza 3 veces al año. Disponible en línea y en CD-ROM, requiere suscripción pagada.

- emedicine (http://www.emedicine.com): requiere inscripción gratuita en el sitio web.

- Principios de Medicina Interna de Harrison (http://www.harrisonsonline.com): disponible en libro de texto y en línea, mediante suscripción pagada.

- Scientific American Medicine (http:// www.samed.com): disponible en línea y en CD-ROM, requiere suscripción pagada.

Encontrar respuesta a las preguntas básicas (o de preparación), muchas veces será insuficiente para tomar decisiones clínicas específicas. Es para responder a las preguntas clínicas (o de acción) cuando necesitamos conocer y adiestrarnos en el uso de sitios más complejos y especializados.

\section{CÓMO RESPONDER NUESTRAS PREGUNTAS CLÍNICAS}

\section{O DE ACCIÓN}

Existen tres tipos de recursos que nos permiten responder a este tipo de preguntas: metabuscadores, bases de datos de revisiones sistemáticas y bases de datos primarias.

Metabuscadores: Son mega sitios que buscan en forma simultánea en múltiples sitios considerados relevantes y relacionados. Son simples de usar y constituyen una forma rápida de encontrar información relevante (búsqueda específica). Los dos metabuscadores más importantes son:

- Tripdatabase (httD://www.tripdatabase.com)

- SumSearch (http://sumsearch.uthscsa.edu/)

Bases de datos de revisiones sistemáticas: Como se describió previamente en esta serie de artículos sobre $\mathrm{MBE}^{5}$, las revisiones sistemáticas constituyen el más alto nivel de evidencia. Los metabuscadores también buscan en estos sitios, por lo que no siempre es necesario acudir directamente a ellos. Constituyen sí, una buena alternativa de búsqueda, especialmente en aquellos temas en que posiblemente existe mucha información.

- Cochrane Library:

(http://www.cochranelibrary.com/cochrane/ abstract.htm): Se puede acceder a los abstracts en forma gratuita. La versión completa (en línea o CD-ROM) requiere suscripción pagada. Existe la alternativa de suscribirse gratuitamente a través del Centro Bireme (www.bireme.br).

- Clinical Evidence:

(http://www.clinicalevidence.com): Agrupación de revisiones sistemáticas y estudios randomizados, que responden a preguntas clínicas específicas. Existe en versión electrónica y como libro editado semestralmente por el British Medical Journal. Requiere suscripción pagada.

Bases de datos primarias: Corresponden a la materia prima para los sitios antes mencionados. Aquí se agrupan los artículos publicados, independientemente de su calidad o relevancia. Utilizar adecuada y eficientemente las bases de datos requiere de entrenamiento, pues de lo contrario, nos entregarán demasiada información (búsqueda sensible), y consumiremos demasiado tiempo. 
Medline constituye la principal base de datos en medicina y es de acceso gratuito a través de su interfase PubMed (http://www.pubmed.gov). Una buena manera de familiarizarse con Medline/ PubMed es realizar alguno de los tutoriales interactivos en línea:

- http://www.nlm.nih.gov/bsd/pubmed_tutorial/ m 1001.html

- http://escuela.med.puc.cl/recursos/mbe2001/ guiadamedline.html

Existen otras bases de datos que pueden servir de complemento en determinados casos (EMBASE, LILACS). Si se tiene el recurso disponible, la incorporación de los servicios que prestan los bibliotecarios es una excelente estrategia para realizar búsquedas eficientes.

\section{ESTRATEGIAS DE BÚSQUEDA ESPECÍFICA EN Medune/PubMed}

Si bien mencionamos la necesidad de adiestramiento para el adecuado uso de Medline/Pubmed, existen algunos consejos simples que permiten realizar búsquedas más específicas.

- Siempre inicie su búsqueda utilizando el buscador de términos Medical Subject Headings, $\mathrm{MeSH}$ (MeSH Database).

\section{REFERENCIAS}

1. http://www.nlm.nih.gov/pubs/factsheets/ medline.html

2. Soto M, Rada G. Formulación de Preguntas en Medicina Basada en la Evidencia. Rev Méd Chile 2003; 131: 1202-3.

3. Richardson WS, WiLSON MC, NishiKaWa J, Hayward RS. The well-built clinical question: a key to
- Utilice límites: Limitar por título en el campo de búsqueda (All fields/Title). En el caso de las preguntas de tratamiento, limitar por tipo de estudio, utilizando metaanálisis o estudio randomizado (Publication types/Metanalysis o Randomized Controlled Trial).

- Utilice el sistema de filtros «clinical queries» (preguntas clínicas): Tiene incorporada una estrategia de búsqueda que responde a nuestros requerimientos.

En el sitio web de la Unidad de Medicina Basada en Evidencia de la Pontificia Universidad Católica (UMBE UC) se encuentran desarrollados todos estos temas en forma más completa, con enlace a los sitios mencionados (http:// escuela.med.puc.cl/recursos/mbe2001).

\section{CONCLUSIÓN}

Para responder a una pregunta clínica o de acción, en forma rápida y efectiva, debemos formularla adecuadamente, y la gran mayoría de las veces realizar una estrategia de búsqueda específica. Como primera elección podemos utilizar un metabuscador, o una base de datos de revisiones sistemáticas, o una combinación de estrategias específicas en Medline/Pubmed.

evidence based decisions. ACP J Club 1995; 123 : A12-3.

4. Haynes RB, Wilczynski N, Mckibbon KA, WaLKer CJ, SinCLAIR JC. Developing optimal search strategies for detecting clinically sound studies in MEDLINE. JAMA 1994; 1: 447-58.

5. Letelier LM, Moore P. Medicina Basada en Evidencia. Visión después de una década. Rev Méd Chile 2003; 131: 939-46. 